\title{
Heartbeat: Balance of safety and efficacy of antihypertensive treatment in elderly patients
}

Hypertension treatment is an essential element in primary prevention of cardiovascular disease (CVD). Despite recent guidelines recommending medical therapy at a lower systolic blood pressure (SBP) threshold, these recommendations remain controversial in the elderly because the balance of safety and efficacy is less clear than in younger adults. In this issue of Heart, Jung and colleagues ${ }^{1}$ used data from the Korean National Insurance sample, including about 420 thousand participants with no prior history of hypertension or CVD, to examine the relationship between a SBP of 130-139 mm $\mathrm{Hg}$ and CVD mortality in three age groups: 40-59 years, 60-69 years and $70-80$ years. In all age groups, there was a positive graded association between SBP and CVD over 10 years of follow-up (figure 1). Each $20 \mathrm{~mm} \mathrm{Hg}$ elevation in SBP was associated with 1.67 -fold (95\% CI 1.57 to 1.78 ) elevated risk for overall CVD mortality in those aged $40-59$ years, 1.40 -fold increase $(95 \%$ CI 1.33 to 1.48 ) in those aged 60-69 years and 1.22-fold increase $(95 \% \mathrm{CI} 1.16$ to 1.29$)$ in those aged $70-80$ years.

In the accompanying editorial, PinhoGomes and Rahimi ${ }^{2}$ discuss the potential limitations of this study and point out that: "harms may outweigh benefits in the elderly or those with multimorbidity, due to higher rates of adverse events and/or because the expected benefit from continuing a preventive medicine is reduced when there is limited life expectancy or high risk of death from competing diseases.' They conclude that "the population-based study by Jung et $a l^{1}$ provides reassurance about an overall positive association between BP and CVD risk in the elderly in a non-Western population. It further highlights the need for more reliable evidence from randomised trials to assess the balance of safety and efficacy of antihypertensive treatment in elderly patients, in particular when BP is not very high and in the presence of comorbidities. Until such evidence becomes available, 'clinical judgement' with its inherent limitations

Correspondence to Professor Catherine M Otto, Division of Cardiology, University of Washington, Seattle,WA 98195, USA; cmotto@uw.edu

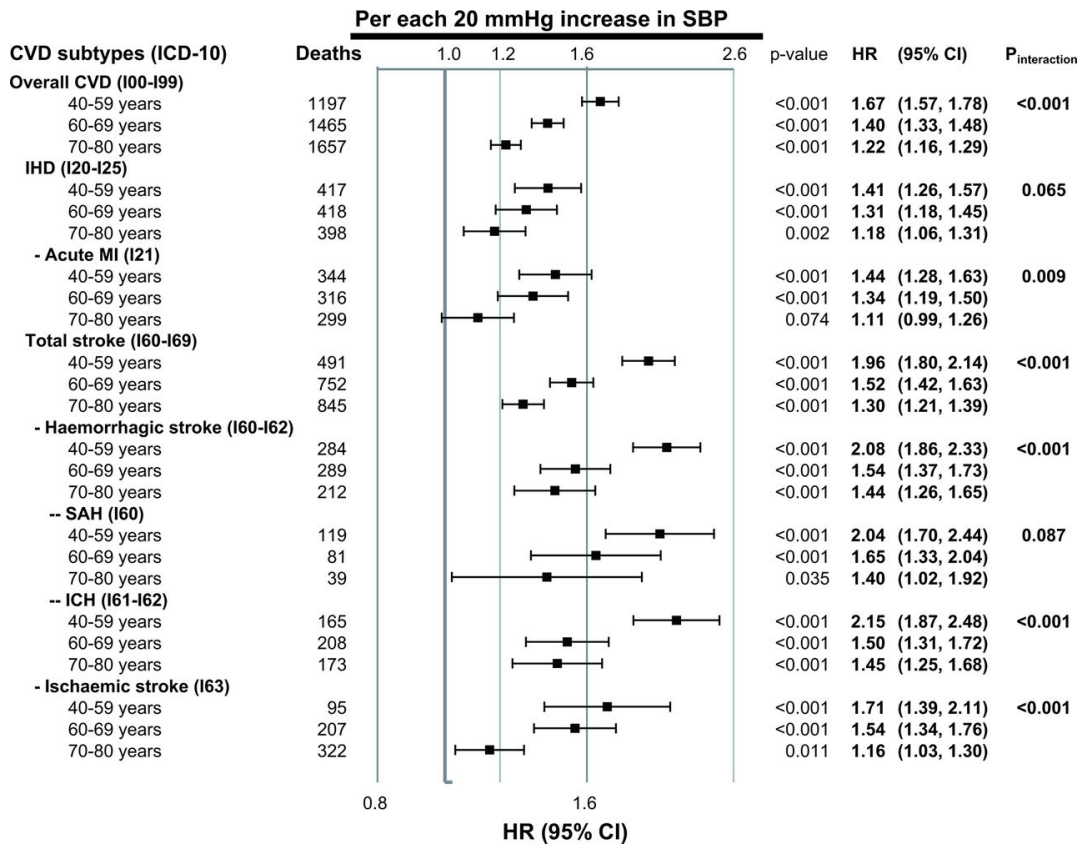

Figure 1 Age-specific effect of per $20 \mathrm{~mm} \mathrm{Hg}$ increase in SBP on CVD mortality. CVD, cardiovascular disease; ICH, intracranial haemorrhage; IHD, ischaemic heart disease; $\mathrm{MI}$, myocardial infarction; SAH, subarachnoid haemorrhage; SBP, systolic blood pressure.

will remain the best standard that guidelines can recommend.'

There is a well-known increased risk of ventricular arrhythmias and sudden cardiac death in adults with mitral valve prolapse (MVP) possibly related to focal or diffuse myocardial fibrosis. Speckle-tracking echocardiography (STE) allows noninvasive measurement of the pattern of ventricular contraction with a heterogenous pattern defined as 'myocardial dispersion'. Ermakov and colleagues ${ }^{3}$ performed a detailed STE study of 32 patients with MVP and complex ventricular ectopy (A-MVP) compared with 27 patients with MVP but no arrhythmic complications (NA-MVP). Despite similar left ventricular ejection fractions and mitral regurgitation severity, there was greater mechanical dispersion in the A-MVP group compared with the NA-MVP group (52 vs $42 \mathrm{~ms}, \mathrm{P}=0.005$ ) (figure 2).

Marra and Basso ${ }^{4}$ point out that the clinical challenge is to identify the small subset of patients with MVP who are at risk of ventricular arrhythmias and sudden cardiac death. Previously proposed arrhythmogenic substrates in patients with MVP include myocardial fibrosis and mitral-annular disjunction with the current study adding mechanical dispersion to this list of possible risk markers. Interestingly, anatomic features of the mitral leaflet and the severity of mitral regurgitation have not been identified as risk factors for ventricular arrhythmias. Marra and Basso ${ }^{4}$ conclude "The current arrhythmic risk stratification of patients with MVP identified by 2D echocardiography include a 12-lead ECG and 24 hours ECG Holter monitoring, but the selection of those patients to address to more invasive and expensive tools such as CMR and electrophysiological study remains to be fully defined.'

A study based on the nationwide Danish registers from 2005 to 2014 suggests that peri-partum cardiomyopathy (PPCM) may be familial. ${ }^{5}$ The prevalence of heart failure in first-degree relatives of 48 women with PPCM was 23\%, compared with $10 \%$ in controls $(P=0.011)$. Auger and colleagues ${ }^{6}$ review the risk factors for PPCM including pre-eclampsia, advanced maternal age, multiple births, and African and Asian descent. Proposed mechanisms of 

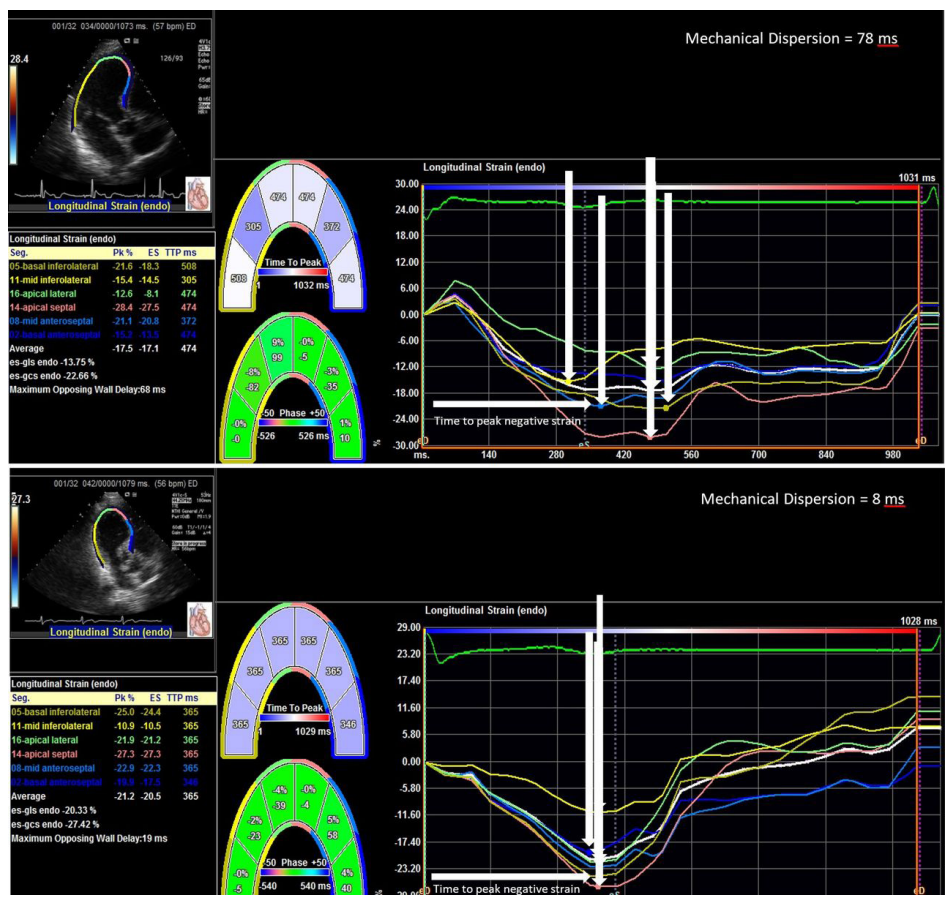

Figure 2 Examples of Tomtec Image Arena measurements of global longitudinal strain (GLS) and time to peak strain (TTP) in arrhythmic mitral valve prolapse (A-MVP) patients (top panel) and non-arrhythmic mitral valve prolapse patients (NA-MVP) (bottom panel). Greater variability in the TTP of each left ventricular (LV) wall segment is seen in the A-MVP patients compared with the NA-MVP patients reflecting increased mechanical dispersion. GLS is defined as the average peak systolic strain for all 18 LV segments. Mechanical dispersion is defined as the SD of the time to peak strain in all $18 \mathrm{LV}$ segments.

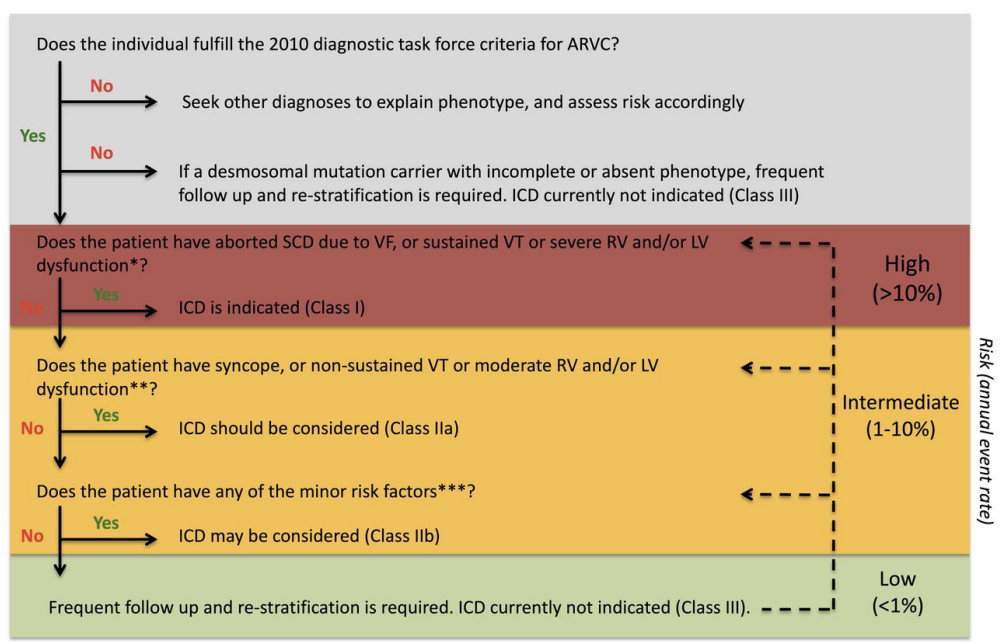

Figure 3 This diagram demonstrates the approach to the risk stratification of patients with arrhythmogenic cardiomyopathies or their mutation positive family members, based on the International Task Force consensus. Based on the annual arrhythmic event rate of more than $10 \%$, between $1 \%$ and $10 \%$ and less than $10 \%$ the high, intermediate and low risk groups are defined. * Severe right ventricular and/or left ventricular dysfunction is defined as RV fractional area change $\leq 17 \%$, or RV ejection fraction $\leq 35 \%$, LV ejection fraction $\leq 35 \%$. * ${ }^{*}$ Moderate left or right ventricular dysfunction is defined as RV fractional area change between $24 \%$ and $17 \%$ or RV ejection fraction between $40 \%$ and $36 \%$ or LV ejection fraction between $45 \%$ and $36 \%$. *** Minor risk factors defined in text. ARVC, arrhythmogenic right ventricular cardiomyopathy; $I C D$, intracardiac cardioverter defibrillator; LV, left ventricle; RV, right ventricle; SCD, sudden cardiac death; VF, ventricular fibrillation; VT, ventricular tachycardia. myocardial dysfunction include abnormal endocrine metabolism, oxidative stress, and genetic associations, particularly with the gene that encodes the sarcomere protein titin. They conclude: "Accurately identifying women at risk of PPCM remains the most likely means of offering appropriate interventions at earlier stages of the disease in order to improve outcomes. It appears likely that the development of a screening tool that incorporates both clinical and biochemical risk markers will provide the best discrimination. In the interim, offering cardiovascular disease screening to first-degree relatives of women with PPCM is likely warranted.'

The Education in Heart article in this issue ${ }^{7}$ provides a concise overview for the diagnosis, risk stratification and management of arrhythmogenic cardiomyopathies, including indications for placement of an implantable defibrillation and family screening (figure 3). A second review article provides a simple approach to the standard ECG to identify the anatomic origin of idiopathic ventricular arrhythmias. ${ }^{8}$

\section{Competing interests None declared.}

Patient consent for publication Not required.

Provenance and peer review Commissioned; internally peer reviewed.

(C) Author(s) (or their employer(s)) 2019. No commercial re-use. See rights and permissions. Published by BMJ.

\section{Check for updates}

To cite Otto CM. Heart 2019;105:1049-1050.

Received 22 May 2019

Accepted 22 May 2019

Heart 2019;105:1049-1050

doi:10.1136/heartjnl-2019-315459

\section{REFERENCES}

1 Jung MH, Yi SW, An SJ, et al. Age-specific associations between systolic blood pressure and cardiovascular mortality. Heart 2019;105:1070-7.

2 Pinho-Gomes AC, Rahimi K. Blood pressure management in the elderly: the need for more randomised evidence. Heart 2019;105:1055-6.

3 Ermakov S, Gulhar R, Lim L, et al. Left ventricular mechanical dispersion predicts arrhythmic risk in mitral valve prolapse. Heart 2019;105:1063-9.

4 Perazzolo Marra M, Basso C. Mechanical dispersion and arrhythmic mitral valve prolapse: substrate and trigger in electrical instability. Heart 2019;105:1053-4.

5 Christiansen MN, Køber L, Torp-Pedersen C, et al. Prevalence of heart failure and other risk factors among first-degree relatives of women with peripartum cardiomyopathy. Heart 2019;105:1057-62.

6 Auger N, Ukah UV, Potter BJ. Peripartum cardiomyopathy: a family affair? Heart 2019;105:1051-2.

7 Protonotarios A, Elliott PM. Arrhythmogenic cardiomyopathies (ACs): diagnosis, risk stratification and management. Heart 2019;105:1117-28.

8 Tzeis S, Asvestas D, Yen Ho S, et al. Electrocardiographic landmarks of idiopathic ventricular arrhythmia origins. Heart 2019;105:1109-16. 